\title{
Increased Photoconductivity Lifetimes in GaAs Nanowires via n-Type and p-Type Shell Doping
}

\author{
Jessica L. Boland ${ }^{1}$, A.Casadei ${ }^{2}$, G. Tütüncouglu ${ }^{2}$, F.Matteini ${ }^{2}$, C. Davies ${ }^{1}$, F.Gaveen ${ }^{2}$, F. Amaduzzi ${ }^{2}$, \\ H.J.Joyce ${ }^{3}$, L.M.Herz ${ }^{1}$, A.Fontcuberta i Morral ${ }^{2}$ and Michael B. Johnston ${ }^{1}$ \\ ${ }^{1}$ Department of Physics, University of Oxford, Oxford, OX1 3PU, United Kingdom \\ ${ }^{2}$ École Polytechnique Fédérale de Lausanne (EPFL), CH-1015 Lausanne, Switzerland \\ ${ }^{3}$ University of Cambridge, Department of Engineering, 9 JJ Thomson Avenue, Cambridge CB3 OFA, United Kingdom
}

\begin{abstract}
Reliable doping in GaAs nanowires is essential for the development of novel optoelectronic devices. Previously, GaAs nanowires have been shown to exhibit extremely short photoconductivity lifetimes of a few picoseconds due to their high surface recombination velocity, which is detrimental for nanowire devices, such as solar cells and nanowire lasers. Here, we show that, by exploiting engineered band-bending via selective doping, this parasitic surface recombination can be reduced. We utilise non-contact time-resolved terahertz spectroscopy to characterise the doping efficiency in n-type and p-type doped GaAs nanowire 8 and show high carrier concentrations of the order of $10^{18} \mathrm{~cm}^{-3}$. The carrier lifetimes were increased by an order of magnitude from 0.13ns for undoped to $3.8 \mathrm{~ns}$ and $2.5 \mathrm{~ns}$ for n-doped and p-doped GaAs nanowires respectively; showing that surface recombination is greatly suppressed as a result of shell doping. We also present a novel effect of p-doping in GaAs nanowires: a rapid decay in photoconductivity within $25 \mathrm{ps}$ after photoexcitation. This fast decay is attributed to rapid electron trapping at the nanowire surface due to doping related band bending. Thus, we demonstrate the advantages of selective doping for enhancement of desirable transport properties in GaAs nanowires, as well as highlighting terahertz spectroscopy as a reliable technique for characterising doped GaAs nanowires ${ }^{1}$.
\end{abstract}

\section{INTRODUCTION}

$\mathrm{H}$ igh surface recombination in GaAs nanowires is a problem in nanowire devices. One way to reduce this surface recombination is by over-coating GaAs nanowires with AlGaAs shells ${ }^{2}$. An alternative approach is to exploit engineered band-bending via selective doping ${ }^{3}$. However, doping incorporation with high carrier concentrations and high mobilities, as well as accurate characterisation of doping effects has proven challenging ${ }^{4}$. To investigate the doping efficiency in GaAs nanowires, undoped, n-type and p-type shell-doped GaAs nanowires were photoexcited with a near-infrared laser at $800 \mathrm{~nm}$ with $35 \mathrm{fs}$ pulses at fluences between 5 and $80 \mu \mathrm{Jcm}^{-2}$. The photoconductivity was measured as a function of frequency and time after photoexcitation. Carrier lifetimes, mobilties and doping concentrations were then extracted from these measurements.

\section{RESULts}

Figure 1 shows the normalized photoconductivity decays for n-doped, p-doped and undoped GaAs nanowires. Through fitting of carrier rate equations, the carrier lifetimes for the ndoped and p-doped GaAs nanowires were determined to be $3.8 \mathrm{~ns}$ and $2.5 \mathrm{~ns}$ respectively, an order of magnitude greater than for the undoped reference sample. The bimolecular recombination constant was also extracted to be $\sim 2 \times 10^{10} \mathrm{~cm}^{3} \mathrm{~s}^{-}$ ${ }^{1}$, coinciding with established values. This demonstrates that non-radiative recombination routes, due to surface states and defects, are suppressed as a result of shell doping.

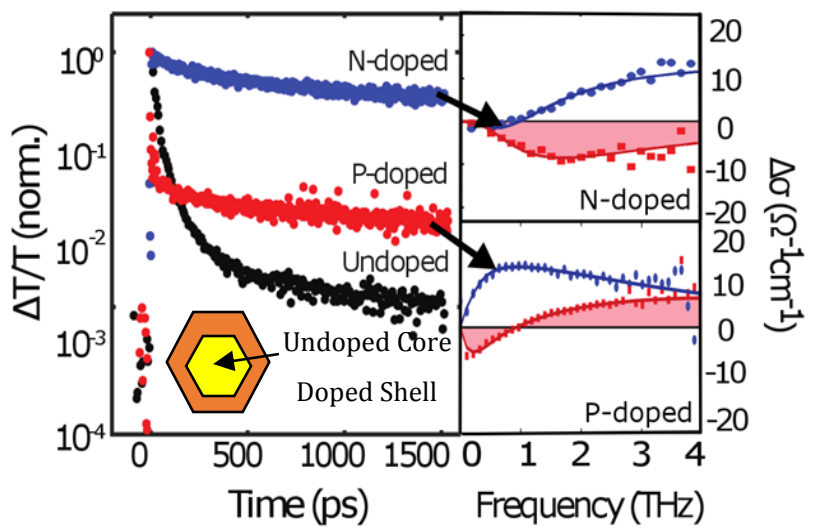

Fig.1: Left: Normalised photoconductivity decay as a function of time for n-doped (blue), p-doped (red) and undoped (black) GaAs nanowires. Right: Photoconductivity spectra for $n$-doped (top) and $\mathrm{p}$ doped (bottom) GaAs nanowires ${ }^{8}$.

For the first time in p-doped GaAs nanowires, a sharp decay in photoconductivity was observed within the first $25 \mathrm{ps}$ after photoexcitation. By examining photoconductivity spectra at different times after photoexcitation, this sharp decay is attributed to rapid electron trapping at the nanowire surface, which leaves a hole-dominated long-lived photoconductivity after $25 \mathrm{ps}$ of photoexcitation.

\section{SUMMARY}

Engineered band bending via n-type and p-type shell doping reduces undesirable surface recombination in GaAs nanowires and enhances photoconductivity lifetimes. Moreover, terahertz spectroscopy offers a reliable method for characterizing doping efficiencies in semiconductor nanowires.

\section{REFERENCES}

[1] J.L.Boland et al., "Increased Photoconductivity Lifetime in GaAs Nanowires by Controlled n-Type and p-Type Doping”, ACS Nano. 10, 42194227 (2016)

[2]. H.J.Joyce, et al., "Electron mobilities approaching bulk limits in surface free GaAs nanowires", Nano Letts.14, 5989-5994 (2014)

[3]Smith, L. M.,et al., "Radiative Recombination in Surface-Free n+/n-/n+ GaAs Homostructures.” Appl. Phys. Lett., 57, 1572-1574 (1990)

[4]. J.L.Boland, et al., "Modulation Doping of GaAs/AlGaAs Core-Shell Nanowires With Effective Defect Passivation and High Electron Mobility", Nano Letts.15,1336-1342 (2015) 